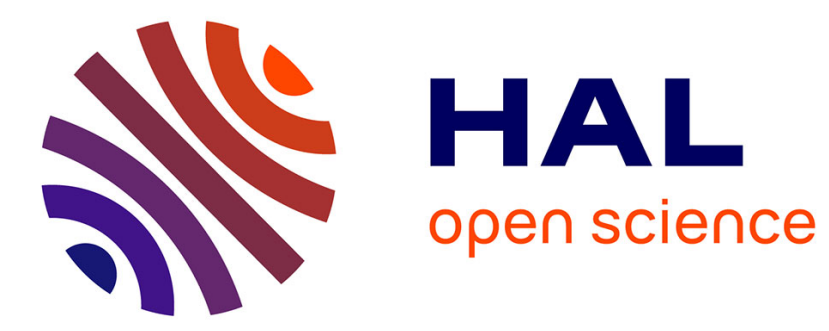

\title{
Elastic behavior of weakly cemented contact
}

Vincent Langlois

\section{To cite this version:}

Vincent Langlois. Elastic behavior of weakly cemented contact. International Journal for Numerical and Analytical Methods in Geomechanics, 2014, to appear, pp.1-7. 10.1002/nag.2342 . hal-01104354

\section{HAL Id: hal-01104354 \\ https://hal.science/hal-01104354}

Submitted on 21 Jan 2015

HAL is a multi-disciplinary open access archive for the deposit and dissemination of scientific research documents, whether they are published or not. The documents may come from teaching and research institutions in France or abroad, or from public or private research centers.
L'archive ouverte pluridisciplinaire HAL, est destinée au dépôt et à la diffusion de documents scientifiques de niveau recherche, publiés ou non, émanant des établissements d'enseignement et de recherche français ou étrangers, des laboratoires publics ou privés. 


\title{
Elastic behavior of weakly cemented contact
}

\author{
V. Langlois* \\ Université Paris-Est, Laboratoire Géomatériaux et Environnement (EA 4508), UPEM, F-77454, Marne-la-Vallée, \\ France
}

\begin{abstract}
SUMMARY
We investigate the elastic behavior of weakly cemented contact. We show that the radial distribution of stresses and the stiffnesses of a cemented contact are governed by the ratio $a / R \Lambda$, where $R, a$ and $\Lambda$ are respectively the grain radius, the contact size, and the ratio of the elastic moduli of cement and grains. Moreover, we show that a cemented contact is always less stiff than a Hertzian contact having a similar size. Finally, we propose accurate approximate expressions of the contact stiffnesses. Copyright (c) 0000 John Wiley \& Sons, Ltd.
\end{abstract}

Received ...

KEY WORDS: Contact cement theory ; contact theory ; granular materials

NOTICE: this is the author's version of a work that was accepted for publication in the International Journal for Numerical and Analytical Methods in Geomechanics. Changes resulting from the publishing process, such as peer review, editing, corrections, structural formatting, and other quality control mechanisms may not be reflected in this document. Changes may have been made to this work since it was submitted for publication. A definitive version was subsequently published in the International Journal for Numerical and Analytical Methods in Geomechanics (http://dx.doi.org/10.1002/nag.2342).

\section{INTRODUCTION}

Cemented dense granular materials are involved in numerous fields including geology $[1,2,3,4,5]$ and civil engineering $[6,7,8]$. Understanding the mechanical behavior of these porous granular materials can provide key insights into phenomena like sedimentary rock failure, landslides, effects on soils behavior of freezing and melting of interstitial water.

Bernabe et al. [2] have studied experimentally the effect of cement on the strength of granular rocks. They observed that cement, even in very small quantity, significantly increases the strength of granular materials as long as the deposition of cement is precisely located at the grain-grain contacts. Alternatively, numerical simulations have been used to study the failure of cemented granular materials. Thus, Bruno et al [1] have shown that strength depends strongly on intergranular bond material properties. More recently, Topin et al. [8] have distinguished different regimes of crack propagation involving particle damage (abrasion or fragmentation) or not (crack propagation either in cement or at the cement-particle interface). They have shown that the particle damage limit

\footnotetext{
${ }^{*}$ Correspondence to: Université Paris-Est, Laboratoire Géomatériaux et Environnement, 5 Boulevard Descartes 77454, Marne-la-Vallée Cedex 2, France 
is controlled by a single parameter combining the cement volume fraction with the grain-cement adhesion.

Likewise, the acoustic and elastic characteristics of cemented granular material may be affected by the properties and structure of intergranular bond material. Within the framework of the effective medium theories (EMT) [9, 10], the elasticity of granular media depends on the normal and tangential contact stiffnesses. Thus, the theoretical description of the elasticity of granular materials has been based on the classical solutions to the problems of normal (Hertz theory, [11]) or oblique $[12,13]$ interaction of elastic spheres. Johnson et al. [14] have studied the compression of two adhesive spheres. Digby [9] solved the problem of interaction of spherical particles that are initially bonded together across small areas. Dvorkin et al. $[15,16]$ have studied the effects on the contact stiffnesses of an elastic intergranular bond (fig. 1a). Their model shows clearly that the cemented contact stiffnesses depend on both the amount of cement and the elastic moduli of cement and grain. Contrary to a Hertzian grain-grain contact, this elastic cement contact model predicts that the contact stiffnesses are independent of the applied pressure. Coupled with EMT, the model reproduces fairly well the experimental results obtained for small amount of cement $[3,17]$.

Although based on the classical contact mechanic and contrary to a Hertzian grain-grain contact, a complete analytical solution of the cemented contact model stays to find, if indeed that is possible. The use of numerical methods is necessary for the calculation of the stiffnesses. To circumvent this difficulty and for practical purposes, statistical approximations of the stiffnesses, having a polynomial form in the ratio $\alpha$ of the contact radius $a$ to the radius of the grains $R$, have been proposed [18,3]: $A \alpha^{2}+B \alpha+C$ where the coefficients, $A, B$ and $C$ depend on the ratio $\Lambda$ of the elastic moduli of cement and grains. However, these expressions lead to an unphysical result: nonzero stiffnesses as the contact size tends to zero. Moreover, their domain of validity is not clearly specified.

In this brief note, we resume the major steps of the cement contact theory (CCT) [16], and show that the ratio $a / R \Lambda$ governs the radial distribution of stresses and the stiffnesses of a cemented contact. New approximate expressions of the normal and tangential stiffnesses are proposed.

\section{NORMAL LOADING OF TWO CEMENTED SPHERES}

At first, we focus on the normal loading of two cemented spheres (figla). We assume that contact stresses and displacements are axisymmetrical with respect to the line that connects the centers of the two spheres. In CCT, the cement layer is assumed to be bonded to the grains, and therefore, no slip or debonding occurs at interfaces between the cement layer and the grains. Thus, the normal displacement of the center of the spherical grain relative to the median plane of the cement layer $\delta_{n}$ can be related to the displacements $\delta_{c}$ of the surface of the cement layer and $\delta_{g}$ of the surface of the grain: $\delta_{c}(r)+\delta_{g}(r)=\delta_{n}$ for any $r$ located inside the contact area $(r<a)$. As the thin cement layer is approximately treated as an elastic foundation [11], the displacement $\delta_{c}$ is proportional to the normal stress $p: \delta_{c}=h(r) p(r) / M_{c}$ where $M_{c}\left(=2 G_{c}\left(1-\nu_{c}\right) /\left(1-2 \nu_{c}\right)\right)$ is the constrained modulus of the cement and $h(r)\left(=R-\sqrt{R^{2}-r^{2}}\right)$ is its initial undeformed thickness. As the cemented contact area is considered small compared with the grain size $(\alpha=a / R \ll 1)$, this thickness can be approximated by $r^{2} / 2 R$, and the displacement $\delta_{g}$ of the surface of the grain is given by $\delta_{g}=\frac{\left(1-\nu_{g}\right)}{\pi G_{g}} \int_{0}^{s_{\max }} \int_{0}^{\pi} p(s, \varphi) d s d \varphi$ [11]. $G_{g}$ and $\nu_{g}$ are respectively the shear modulus and the Poisson's ratio of the grains. The parameters, $s$ and $\varphi$, give the position of a point inside the domain of integration $S$ defined on figure $1 \mathrm{~b}$. However, under this formulation, the domain of integration depends on the contact size $a$. Thus, to find the governing parameters, we introduce normalized coordinates $s^{\prime}=s / a$ and $r^{\prime}=r / a$. The displacement $\delta_{g}$ is now given by $\delta_{g}=\frac{\left(1-\nu_{g}\right) a}{\pi G_{g}} \iint_{S^{\prime}} p\left(s^{\prime}, \varphi\right) d s^{\prime} d \varphi$ and the new domain of integration $S^{\prime}$ is independent of the contact size. 


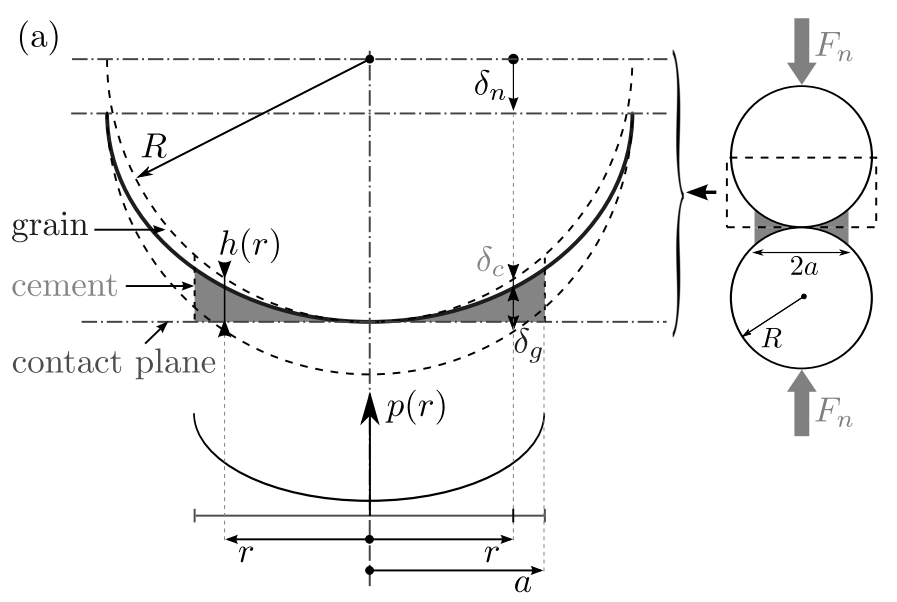

(b)

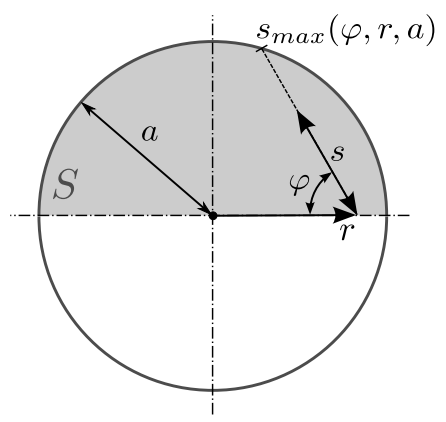

Figure 1. (a) Cross-section of a cemented grain before and after loading (b) A cemented contact region. The domain of integration $S$ corresponds to the shaded area.

By combining the above equations and by normalizing the radial distribution of the normal stress $P\left(r^{\prime}\right)=\frac{\alpha^{2}}{M_{c}} p\left(r^{\prime}\right)$, we arrive at the following integral equation:

$$
\frac{\Lambda_{n}}{\alpha} \iint_{S^{\prime}} P\left(s^{\prime}, \varphi\right) d s^{\prime} d \varphi+\frac{1}{2} r^{\prime 2} P\left(r^{\prime}\right)=\frac{\delta_{n}}{R}
$$

where $\Lambda_{n}=\left(1-\nu_{g}\right) M_{c} / \pi G_{g}$.

Then, by dividing both sides of equation 1 by $\delta_{n} / R$ and, by putting $P_{1}=\frac{P}{\delta_{n} / R}$, we obtain:

$$
\frac{\Lambda_{n}}{\alpha} \iint_{S^{\prime}} P_{1}\left(s^{\prime}, \varphi\right) d s^{\prime} d \varphi+\frac{1}{2} r^{\prime 2} P_{1}\left(r^{\prime}\right)=1
$$

As no evident analytical solution of the equation 2 can be found, a numerical method must be used. However, by considering the equation, its solution is a function of both the position $r^{\prime}$ and the ratio $\alpha / \Lambda_{n}$, and consequently, the solution of the equation 1 , given by $P=P_{1}\left(r^{\prime}, \frac{\alpha}{\Lambda_{n}}\right) \cdot \frac{\delta_{n}}{R}$, is proportional to the displacement $\delta_{n}$. Therefore, the contact-cement model leads to a linear elasticity totally different from the non linear elasticity of a Hertzian contact between two uncemented spheres [11]. As we will discuss further later on, the contact-cement model predicts such a linear elasticity as long as the cement behavior is linear elastic and the size of the cemented contact size is constant $[19,20]$.

Another major result of the contact-cement model concerns the radial distribution of stresses. As we have shown before, the ratio $\alpha / \Lambda_{n}$ determines the radial distribution of the normal stress (fig 2a): the maximal stress is located at the center of the contact when $\alpha / \Lambda_{n} \gg 1$ ("soft cement"), or at the periphery when $\alpha / \Lambda_{n} \ll 1$ ("stiff cement"). The threshold ratio between both regimes is around 2 . In the stiff cement regime, the radial distribution of the normal stress tends to the one 

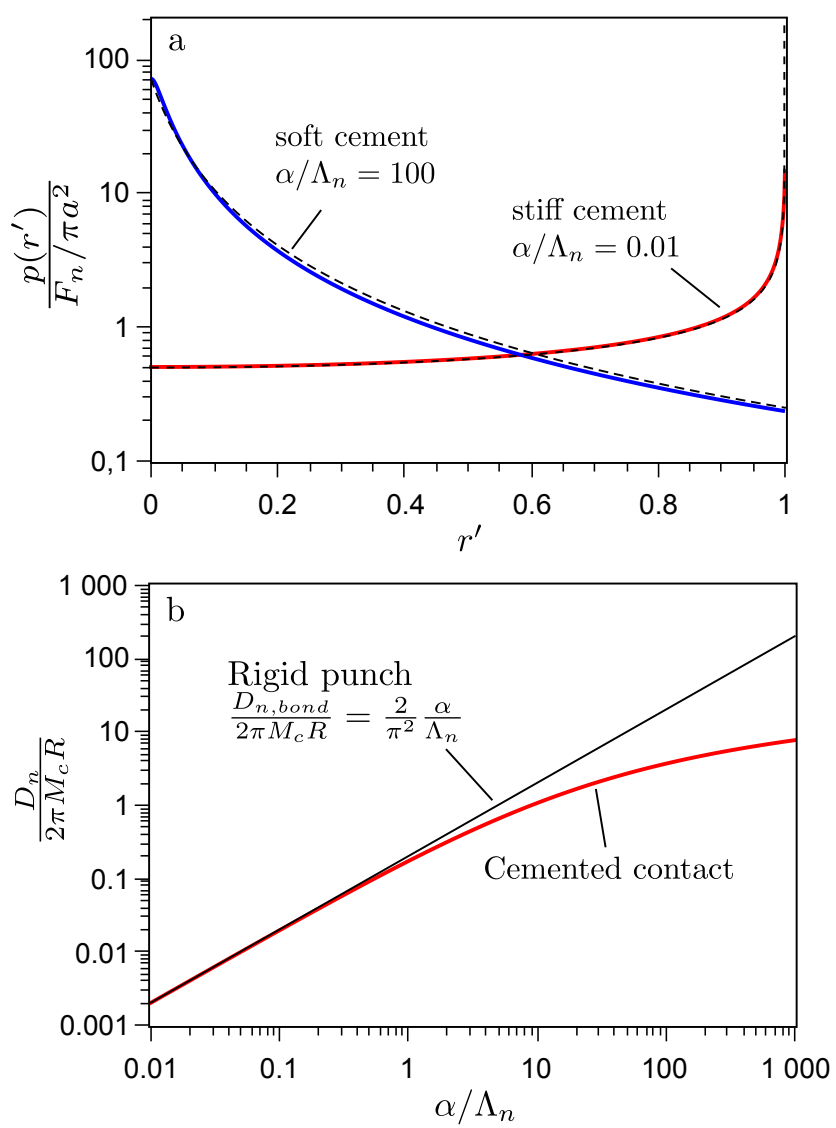

Figure 2. Contact-cement model predictions: (a) radial distribution of normal stresses in two cases: soft cement and stiff cement. The dashed curves correspond to asymptotic solutions: $1 / 2\left(1-r^{\prime 2}\right)^{1 / 2}$ for stiff cement, and $2 /\left(r^{\prime}+r_{c}^{\prime}\right)^{2}$ for soft cement (b) Cemented contact stiffness as function of contact size $\alpha$.

obtained by the indentation of a circular rigid flat punch over an elastic half-space $p_{0}\left(1-r^{\prime 2}\right)^{-1 / 2}$ [11]. In the soft cement regime, the radial distribution of the normal stress is close to $2 /\left(r^{\prime}+r_{c}^{\prime}\right)^{2}$ with $r_{c}^{\prime}=2 \pi \Lambda_{n} / \alpha$.

As the elasticity of a cemented contact is linear, the normal stiffness $D_{n}$ is calculated by dividing the normal force $F_{n}\left(=\int_{0}^{a} p(r) .2 \pi r d r\right)$ by the displacement $\delta_{n}$. We find that the normal stiffness can be expressed as $D_{n}=2 \pi M_{c} k_{n} R$ with $k_{n}=\int_{0}^{1} P_{1}\left(r^{\prime}\right) r^{\prime} d r^{\prime}$. Figure $2 \mathrm{~b}$ shows the nonlinear relationship between the normal stiffness and the contact radius. In the "stiff cement" regime, the normal stiffness tends to the one obtained by the action of a rigid flat punch $D_{n, \text { rigid }}=$ $4 a G_{g} /\left(1-\nu_{g}\right)$. This result is quite remarkable, because the expression of the stiffness $D_{n, \text { rigid }}$ is identical to the one obtained in a Hertzian sphere-sphere contact. Therefore, as $D_{n}<D_{n, \text { rigid }}$, a cemented contact is always less stiff than a sphere-sphere contact having a similar size.

To avoid the use of numerical method, the normal stiffness of a cemented contact $D_{n}$ can be expressed as $D_{n}=D_{n, \text { rigid }} /\left(1+f\left(\alpha / \Lambda_{n}\right)\right)$. The values of the corrective function $f$ can be evaluated, with a relative error from the results of the numerical method less than $\pm 0.1 \%$ in the range of 0 to 1000 for $\alpha / \Lambda_{t}$, using:

$$
f(x)=0.3092 x^{0.9098} \frac{0.1036 x^{0.4139}+1}{x^{0.4139}+1}
$$

Figure 3 compares the normal stiffnesses calculated by using both approximations, the one given by $[18,3]$ and the one proposed in this paper. The domain of validity of Dvorkin and Nur's approximate expression appears restricted to values of $\Lambda_{n}$ close to 1 , namely when the elastic moduli 


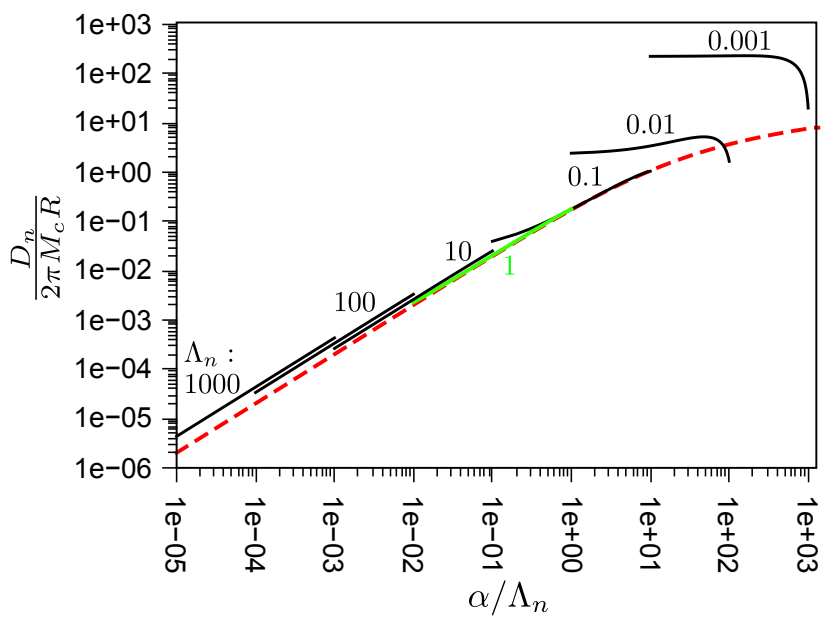

Figure 3. Comparison of the approximate stiffnesses: this paper (dashed line), from [18] for various values of $\Lambda_{n}$ (see curves) and $\alpha$ varying from 0.01 to 0.5 (plain lines).

of cement and grains are similar. In all others cases, even in the stiff cement regime, their expression is inaccurate to determine the normal stiffness.

As mentioned before, the pure elastic model for a cemented contact predicts that the contact stiffness is independent of the applied pressure because of the constant area of an elastic cemented contact [16]. However, for two elastic spheres uniformly coated with a thin elastic cement layer, the contact stiffness may increase when the spheres are axially compressed together, due to the increasing contact area $[19,21]$. For two cemented grains with a plastic behavior of the cement layer, two pressure-activated mechanisms can occur and increase the contact stiffness [20, 17]: an increase of the contact size due to the plastic flow of the cement towards the periphery of the contact area, and the formation of a direct grain-grain contact stiffer than a cemented contact. On the contrary, some pressure-activated mechanisms could lead to the decrease of the contact stiffness [17]: debonding/fracture at the cement-grain interface or inside the cement.

\section{TANGENTIAL LOADING OF TWO CEMENTED SPHERES}

Dvorkin et al [16] extend the method used in the normal loading to the case of tangential loading. Here, the problem is to find the radial distribution of normalized shear stresses $T\left(r^{\prime}\right)=\frac{\alpha^{2}}{G_{c}} t\left(r^{\prime}\right)$ which is solution of the following equation:

$$
\left.\frac{\Lambda_{t}}{\alpha} \iint_{S^{\prime}}\left(1-\nu_{g} \sin ^{2} \varphi\right)\right) T d s^{\prime} d \varphi+\frac{1}{2} r^{2} T=\frac{\delta_{t}}{R}
$$

where $\delta_{t}$ represents the tangential displacement of the center of the grain from the center of the contact area. By considering the equation 3 , we find that the radial distribution of normalized shear stresses is determined by the Poisson's ratio of the grains $\nu_{g}$ and the ratio $\alpha / \Lambda_{t}$ where $\Lambda_{t}=G_{c} / \pi G_{g}$. As previously, the model leads to a linear tangential stiffness of the cemented contact given by: $D_{t}=2 \pi G_{c} k_{t} R$ where $k_{t}=\int_{0}^{1} T_{1}\left(r^{\prime}\right) r^{\prime} d r^{\prime}$ and $T_{1}$ is the solution of the equation 3 for $\delta_{t} / R=1$. Moreover, the main results obtained with a normal loading and concerning the radial distribution of stresses are still valid for a tangential loading.

A method similar to the one used in normal loading could be used to solve the equation 3 . However, in developing the term $1-\nu_{g} \sin ^{2}(\varphi)$, we arrive at: 


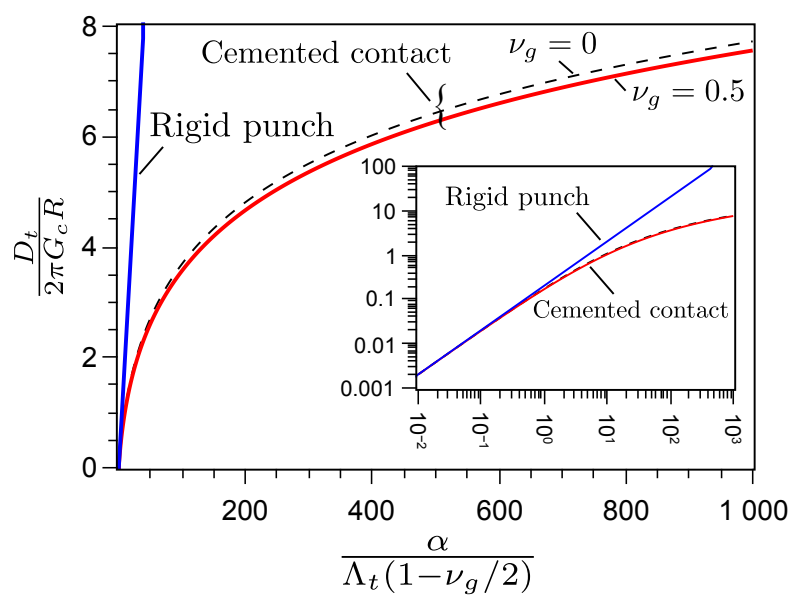

Figure 4. Contact-cement model predictions: tangential stiffness as function of the parameter $\alpha / \Lambda_{t}\left(1-\nu_{g} / 2\right)$. The inset corresponds to the same curves in a log-log plot.

$$
\left[\frac{\Lambda_{t}}{\alpha}\left(1-\frac{\nu_{g}}{2}\right) \iint T\left(s^{\prime}, \varphi\right) d s^{\prime} d \varphi+\frac{1}{2} r^{\prime 2} T\left(r^{\prime}\right)\right]+\left[\frac{\Lambda_{t}}{\alpha} \frac{\nu_{g}}{2} \iint_{S^{\prime}} \cos (2 \varphi) T\left(s^{\prime}, \varphi\right) d s^{\prime} d \varphi\right]=\frac{\delta_{t}}{R}
$$

The terms contained in the first bracket are similar to the left-side terms of the equation 1 by substituting $\Lambda_{n} / \alpha$ for $\left(1-\frac{\nu_{g}}{2}\right) \Lambda_{t} / \alpha$. Due to the zero mean value of $\cos (2 \varphi)$ on the range $[0, \pi]$, the terms in the second bracket are small compared to the first terms and correspond to a corrective term. Therefore, the radial distribution of shear stresses and the tangential stiffness are meanly determined by the ratio $\alpha / \Lambda_{t}\left(1-\nu_{g} / 2\right)$. Figure 4 illustrates this result. Moreover, as shown in the inset of figure 4, the tangential stiffness tends to the one obtained by the action of a rigid flat punch $D_{t, \text { rigid }}=8 a G_{g} /\left(2-\nu_{g}\right)$ in the "stiff cement" regime $\left(\alpha / \Lambda_{t} \ll 1\right)$. Again, since this expression of the tangential stiffnesses and the one of a sphere-sphere contact in a no-slip condition [11] are identical, a cemented contact is always less stiff than a bonded sphere-sphere contact having a similar size.

Finally, the tangential stiffness of a cemented contact can be approximate by:

$$
D_{t, 0}=D_{t, \text { rigid }} /\left(1+f\left(\alpha / \Lambda_{t}\left(1-\nu_{g} / 2\right)\right)\right)
$$

with a relative error from the results of the numerical method less than $6 \%$ in the range of 0 to 1000 for $\alpha / \Lambda_{t}$. A better approximation $(<0.2 \%)$ is obtained by:

$$
D_{t}=\left(1+\frac{\nu_{g}}{2-\nu_{g}} g\left(\alpha / \Lambda_{t}\left(1-\nu_{g} / 2\right)\right)\right) D_{t, 0}
$$

where $g(x)=-0.428 x /\left(1+1.336 x^{1.222}+4.031 x^{0.523}\right)$.

\section{CONCLUSION}

Starting from the equations of CCT, we show that radial distribution of the stresses and the stiffnesses of a cemented contact are governed by the ratio $a / R \Lambda$, where $R, a$ and $\Lambda$ are respectively the grain radius, the contact size, and the ratio of the elastic moduli of cement and grains. In the "rigid cement" regime $(a / R \Lambda<<1)$, the stiffnesses of a cement contact converges to the stiffnesses of a Hertzian contact having a similar size. Therefore, when $a / R \Lambda<0.1$, both contact models almost predict the same stiffnesses with a difference less than 3\%. Moreover, we show that a cemented contact is always less stiff than a Hertzian contact having a similar size. Consequently, the 
stiffnesses of both contact models are upper (cement contact) and lower (Hertzian contact) bounds of the stiffnesses of prestressed cement contact.

\section{REFERENCES}

1. Bruno, M.S., Nelson, R.B., Microstructural analysis of the inelastic behavior of sedimentary rock, Mech. of Mater, 1991; 12, 95-118.

2. Bernabe, Y., Fryer D.T., Hayes, J.A., The effect of cement on the strength of granular rocks, Geophys. Res. Lett. 1992; 19, 1511-1514.

3. Dvorkin, J., Berryman, J., Nur, A., Elastic moduli of cemented sphere packs. Mechanics of Materials 1999; 31, 461-469.

4. Chand, S., Minshull, T., Gei, D., Carcione, J., Elastic velocity models for gas-hydrate-bearing sediments - a comparison Geophys. J. Int. 2004; 159, 573-590.

5. García, X., Medina, E., Acoustic response of cemented granular sedimentary rocks: molecular dynamics modeling. Phy. Rev. E 2007; 75, 061308.

6. Benhamida, A., Bouchelaghem, F., Dumontet, H., Effective properties of a cemented or an injected granular material. Int. J. Numer. Anal. Meth. Geomech. 2005; 29, 187-208.

7. DeJong, J., Fritzges, M., Nüsslein, K., Microbially induced cementation to control sand response to undrained shear. J. Geotech. Geoenviron. Eng. 2006; 132, 1381-1392.

8. Topin, V., Delenne, J.-Y., Radjaï, F., Brendel, L., Mabille, F., Strength and failure of cemented granular matter. Eur. Phys. J. E 2007; 23, 413-429.

9. Digby, P., The effective elastic moduli of porous granular rocks. J. Appl. Mech. 1981; 48, 803-808.

10. Winkler, K., Contact stiffness in granular porous materials: comparison between theory and experiment. Geophys. Res. Lett. 1983; 10, 1073-1076.

11. Johnson, K. L., Contact Mechanics. Cambridge Univ. Press 1989, Cambridge.

12. Mindlin, R.D., Compliance of Elastic Bodies in Contact. J. Appl. Mech. 1949;71, 259-268

13. Walton, K., The oblique compression of two elastic spheres J. Mech. Phys. Solid 1978;26, 139-150

14. Johnson, K. L., Kendall K., Roberts, A. D., Surface Energy and the Contact of Elastic Solids Proc. R. Soc. Lond. A $1971 ; 324,301-313$

15. Dvorkin, J., Mavko, G., Nur, A. The effect of cementation on the elastic properties of granular material. Mechanics of Materials 1991; 12, 207-217.

16. Dvorkin, J., Nur, A., Yin, H. Effective properties of cemented granular materials. Mechanics of Materials 1994; 18, 351-366.

17. Langlois, V., Jia, X., Acoustic probing of elastic behaviour and damage in weakly cemented granular media. Phy. Rev. E 2014; 89, 023206.

18. Dvorkin, J., Nur, A., Elasticity of high-porosity sandstones: Theory for two north sea data sets. Geophysics 1996; 61, 1363-1370.

19. Elata, D., Dvorkin, J., Pressure sensitivity of cemented granular materials. Mechanics of Materials 1996; 23(2), 147-154.

20. Dvorkin, J., Yale, D., Plastic compaction of cemented granular materials. Computers and Geotechnics 1997; 20, 287-302.

21. de Gennes, P.-G., Static compression of a granular medium: the "soft shell" model. Europhys. Lett. 1996; 35, 145149. 